\title{
The influence of biological sex and sex hormones on bile acid synthesis and cholesterol homeostasis
}

\author{
Taylor Phelps, Erin Snyder, Erin Rodriguez, Hailey Child and Pamela Harvey* (1)
}

\begin{abstract}
Obesity and elevated serum lipids are associated with a threefold increase in the risk of developing atherosclerosis, a condition that underlies stroke, myocardial infarction, and sudden cardiac death. Strategies that aim to reduce serum cholesterol through modulation of liver enzymes have been successful in decreasing the risk of developing atherosclerosis and reducing mortality. Statins, which inhibit cholesterol biosynthesis in the liver, are considered among the most successful compounds developed for the treatment of cardiovascular disease. However, recent debate surrounding their effectiveness and safety prompts consideration of alternative cholesterol-lowering therapies, including increasing cholesterol catabolism through bile acid (BA) synthesis. Targeting the enzymes that convert cholesterol to BAs represents a promising alternative to other cholesterol-lowering approaches that treat atherosclerosis as well as fatty liver diseases and diabetes mellitus. Compounds that modify the activity of these pathways have been developed; however, there remains a lack of consideration of biological sex. This is necessary in light of strong evidence for sexual dimorphisms not only in the incidence and progression of the diseases they influence but also in the expression and activity of the proteins affected and in the manner in which men and women respond to drugs that modify lipid handling in the liver. A thorough understanding of the enzymes involved in cholesterol catabolism and modulation by biological sex is necessary to maximize their therapeutic potential.
\end{abstract}

Keywords: Bile, Cholesterol, Nuclear receptors, Estrogen, Hormones, Cytochrome P450

\section{Background}

Bile acids (BAs) are synthesized from cholesterol in liver hepatocytes and are secreted into the small intestine to emulsify and promote absorption of dietary lipids [1]. Approximately 95\% of BAs are reabsorbed by the intestinal epithelium and returned to the liver via the portal vein [2]. The remaining $5 \%$ of the total BA pool is excreted daily and replaced by hepatic de novo cholesterol synthesis [1,2]. A small percentage of BAs is not immediately recycled, and these have recently been identified as systemic signaling molecules with important roles in glucose and lipid homeostasis [2].

\section{Sexual dimorphisms in BA synthesis and excretion}

Although many roles have been described for BAs with regard to cholesterol homeostasis and endocrine signaling

* Correspondence: pamela.harvey@colorado.edu

Department of Molecular, Cellular, and Developmental Biology, University of Colorado at Boulder, Boulder, CO 80309, USA in both hepatic and extrahepatic tissues, we focus this review on conversion of cholesterol to BAs and sexual dimorphisms in the activity and regulation of enzymes involved in this process. In mice and humans, the rate of BA synthesis and BA pool composition are sexually dimorphic [3]. Wild-type female mice, for example, have a larger total BA pool than male mice; however, females excrete less fecal BA and catabolize less cholesterol via BA production than males [3-5]. Age-related differences in hormone levels are implicated in the differential production of BA in females [6]. Systemic cholesterol homeostasis is achieved by its synthesis and conversion to BAs in the liver as well as feedback mechanisms mediated by BAs. Consideration of sexual dimorphisms in BA synthesis is a critical complement to known modulation of cardiovascular and hepatic diseases by biological sex.

(c) The Author(s). 2019 Open Access This article is distributed under the terms of the Creative Commons Attribution 4.0 International License (http://creativecommons.org/licenses/by/4.0/), which permits unrestricted use, distribution, and reproduction in any medium, provided you give appropriate credit to the original author(s) and the source, provide a link to the Creative Commons license, and indicate if changes were made. The Creative Commons Public Domain Dedication waiver (http://creativecommons.org/publicdomain/zero/1.0/) applies to the data made available in this article, unless otherwise stated. 


\section{Synthesis of BAs in the liver}

Cytochrome P450s (CYPs) comprise the majority of the estimated 17 enzymes involved in BA synthesis, with abnormalities in their expression or function leading to liver, digestive, and systemic pathologies secondary to elevated cholesterol [1, 7]. CYPs convert 27-carbon (C27) cholesterol to 24-carbon (C24) BAs that are characterized by a carboxylated side chain (carbons 20-24) and hydroxyl groups at various positions on the steroid core (carbons 1-19) (Table 1) [1]. Although two pathways are responsible for their production, $75 \%$ of the total BA pool is produced by the classical (neutral) pathway. The production and ratio of the BAs cholic acid (CA) and chenodeoxycholic acid (CDCA), the major BA species in humans, are also mediated by the classical pathway [8]. By contrast, the alternative (acidic) pathway completes side chain oxidation prior to modification of the steroid ring and results in production of CDCA only [9]. The yield of the alternative pathway is significantly smaller than that of the classical pathway and varies between species; in humans, the alternative pathway produces approximately $10 \%$ of the total BAs to replace those excreted from the intestines [10]. Nevertheless, the activity of alternative pathway enzymes can be upregulated by excess cholesterol or with hepatic pathology, and using enzymes shared with the classical pathway enzymes, can produce both CA and CDCA [11].

\section{Of mice or men}

Although mouse models have been critical in identifying the roles of enzymes in the BA synthetic pathways, it is important to note features that distinguish humans from rodents in this regard. Notably, in mice, the yield of the classical pathway represents approximately $60 \%$ of the total BAs [12], whereas in humans, this pathway is responsible for $90 \%$ of BA synthesis [9]. Additionally, BA species are more variable in mice and include muricholic acids that are not present in healthy humans [13]. Despite these differences, mouse models displaying dysfunction of the classical pathway show significant attenuation in the excretion of fecal BAs similar to that of humans [8]. Where appropriate, we indicate sexual dimorphisms in human and rodent studies throughout the text and conclude with a review of the role of sex hormones in regulation of the genes involved in BA synthesis (Tables 2 and 3). The role and respective sex differences of critical CYPs that participate in the synthesis and metabolism of sex hormones are not considered here but have been reviewed extensively elsewhere [14].

\section{Enzymes of the classical and alternative bile acid synthesis pathways}

There are approximately 17 enzymes involved in BA synthesis in the liver. Each is regulated by complex networks that involve both cholesterol and BAs as well as by signaling mediated by sex hormones. We limit our review to highlight those enzymes that (1) have critical roles in BA synthesis that when disrupted lead to clinical pathology in humans and (2) have evidence for regulation by biological sex or sex hormones. Enzymes that lack sexual dimorphisms or regulation by sex hormones are not included in this review but are reviewed elsewhere $[2,7]$. For example, although oxysterol $7 \alpha$-hydroxylase (CYP7B1) is an integral enzyme in the alternative pathway of BA synthesis, disruption of the gene causes no significant pathology in the liver and cholesterol homeostasis is normal. For each enzyme section, we begin with a brief summary of the function of the enzyme and regulation of its expression followed by a description of the phenotype resulting from experimental manipulation of the gene (for complete review see [2, 7]). Each section concludes

Table 1 Major classical and alternative pathway enzymes with subcellular location, reaction type and position of structure modification, and product(s) formed

\begin{tabular}{|c|c|c|c|c|}
\hline Major enzymes & Localization & Reaction & Position on Cholesterol & Product \\
\hline \multicolumn{5}{|l|}{ Classical pathway } \\
\hline CYP7A1 & Endoplasmic Reticulum & Sterol ring modification & $C 7$ & 7a-hydroxycholesterol \\
\hline AKR1D1 & Cytosol & Sterol ring modification & C5 & 5b-reduced intermediates \\
\hline CYP8B1 & Endoplasmic Reticulum & Sterol ring modification & $\mathrm{C} 12$ & 7a, 12a-dihydroxy-4-cholestan-3-one \\
\hline CYP27A1 & Mitochondria & Side chain modification & $\mathrm{C} 27$ & 27-hydroxycholesterol \\
\hline CYP3A4 & Endoplasmic Reticulum & Sterol ring modification & C4 & 4alpha-hydroxy-cholesterol \\
\hline \multicolumn{5}{|c|}{ Alternative pathway } \\
\hline CYP27A1 & Mitochondria & Side chain modification & $\mathrm{C} 27$ & $\begin{array}{l}\text { 27a-hydroxylcholesterol 25a-hydroxycholesterol } \\
24 a-h y d r o x y c h o l e s t e r o l\end{array}$ \\
\hline CYP7B1 & Endoplasmic Reticulum & Sterol ring modification & $\mathrm{C7}$ & 7a-hydroxylated intermediates \\
\hline AKR1D1 & Cytosol & & & 5b-reduced intermediates \\
\hline CYP27A1 & Mitochondria & Side chain modification & $\mathrm{C} 27$ & CDCA \\
\hline
\end{tabular}


Table 2 Sexual dimorphisms in mice lacking enzymes that participate in formation of bile acids with clinical phenotypes in humans (differences between sexes not considered in these studies)

\begin{tabular}{lll}
\hline Bile Acid Synthetic Enzyme & Phenotype in knockout in terms of BAs \& cholesterol & Human phenotype when gene is mutated \\
\hline CYP7A1 & $\begin{array}{l}\text { Lithogenic composition of gallstones with increased dietary } \\
\text { cholesterol in females [30] }\end{array}$ & Statin-resistant hypercholesterolemia [28] \\
& BA pool - larger in females [30] & \\
& BA pool composition - higher CA in females [30] & \\
& Hepatic cholesterol accumulation with increased dietary & 94\% reduction in fecal BA excretion [28] \\
& cholesterol in females (males not reported) [31] & \\
& Maternal consumption of high fat diet results in male & Premature atherosclerosis [28] \\
& Offspring with lower expression than females [33] & \\
BA pool increases in male more dramatically than in females [3] & \\
& $\begin{array}{l}\text { Greater compensatory response by CYP7A1 in female knockout, } \\
\text { resulting in increased CDCA [3] }\end{array}$ & \\
CYP27A1 & Sex differences not reported [55, 59] & Cerebrotendinous xanthomatosis [55] \\
ARK1D1 & Higher hepatic BA concentration and lean phenotype in males [69] & \\
CYP3A4 & Sex differences not reported in genome-edited rats [75] & \\
\hline
\end{tabular}

with a review of the sexual dimorphisms in enzyme expression or function as well as resulting phenotypes.

\section{CYP7A1}

The first enzyme in the classical pathway, cholesterol $7 \alpha$-hydroxylase (CYP7A1), catalyzes the rate-limiting step of BA synthesis from cholesterol [15] (Fig. 1). CYP7A1 hydroxylates the $7 \alpha$-position on cholesterol to produce $7 \alpha$-hydroxycholesterol (Table 1 ). Due to its importance in regulating the rate of BA synthesis, its expression is tightly regulated by a complex mechanism involving cholesterol and BA interactions with nuclear receptors. Transcription is promoted by interactions between cholesterol and the cholesterol-sensor liver $\mathrm{X}$ receptor alpha $(\mathrm{LXR} \alpha)$ and is negatively regulated by BAs via interaction with farnesoid X receptor (FXR) $[17,18]$. $\mathrm{BA}$ activation of FXR induces expression of the orphan nuclear receptor small heterodimer partner (SHP), which then interacts with liver receptor homolog-1 (LRH1) to inhibit CYP7A1 expression via its BA response element (BARE) $[19,20]$. BAs in the intestines indirectly inhibit
CYP7A1 expression by promoting expression of fibroblast growth factor (FGF) 15/19, which is released and binds to hepatic FGF receptor 4 (FGFR4). Subsequent activation of c-Jun N-terminus kinase (JNK) signaling inhibits production of the CYP7A1 transcript [20, 21].

The BA pool in mice lacking Cyp7a1 is approximately $60 \%$ of the size of wild-type mice with a lower concentration of CA [15]. Although BAs are amphipathic molecules, the relationship between the hydrophilic $\alpha$-side defined primarily by the presence of hydroxyl groups, and of the hydrophobic $\beta$-side defined by methyl groups, contributes significantly to the efficiency of sterol solubilization in the intestines [22]. For example, CA and CDCA, both of which are classified as hydrophobic BAs, solubilize sterols at a higher rate in the intestines and regulate expression of genes involved in BA synthesis more efficiently than those that are hydrophilic [23-26]. Reduction in these BAs significantly reduces the uptake of sterols from the intestines; therefore, genetic removal of Cyp $7 a 1$ promotes increased intestinal sterol synthesis and increases 12alpha-hydroxylase (Cyp8b1) expression aimed at

Table 3 Regulation of enzymes involved in bile acid synthesis by hormones and their respective hormone receptors

\begin{tabular}{llll}
\hline Enzyme & Hormone/receptor & Regulation & Reference \\
\hline CYP7A1 & 17alpha-ethynylestrodrial (EE2)/ER-alpha & Downregulates CYP7A1 expression & [123] \\
& GPR30 & Upregulates CYP7A1 expression & [113] \\
CYP8B1 & 17alpha-ethynylestrodrial (EE2)/ER-alpha & Downregulates CYP8B1 expression & [123[ \\
& Estrogen (with biliary diversion, not intact enterohepatic circulation) & Downregulates CYP8B1 expression & {$[124]$} \\
CYP27A1 & Estrogen/ER-alpha \& ER-beta & Downregulates CYP27A1 expression & [122] \\
& Androgens/androgen receptor & Upregulates CYP27A1 expression & [122] \\
CYP3A4 & Estrogen & Downregulates CYP3A4 & [114] \\
AKR1D1 & Testosterone & Inhibits upregulation of AKR1D1 by Estrogen & [114] \\
\hline
\end{tabular}




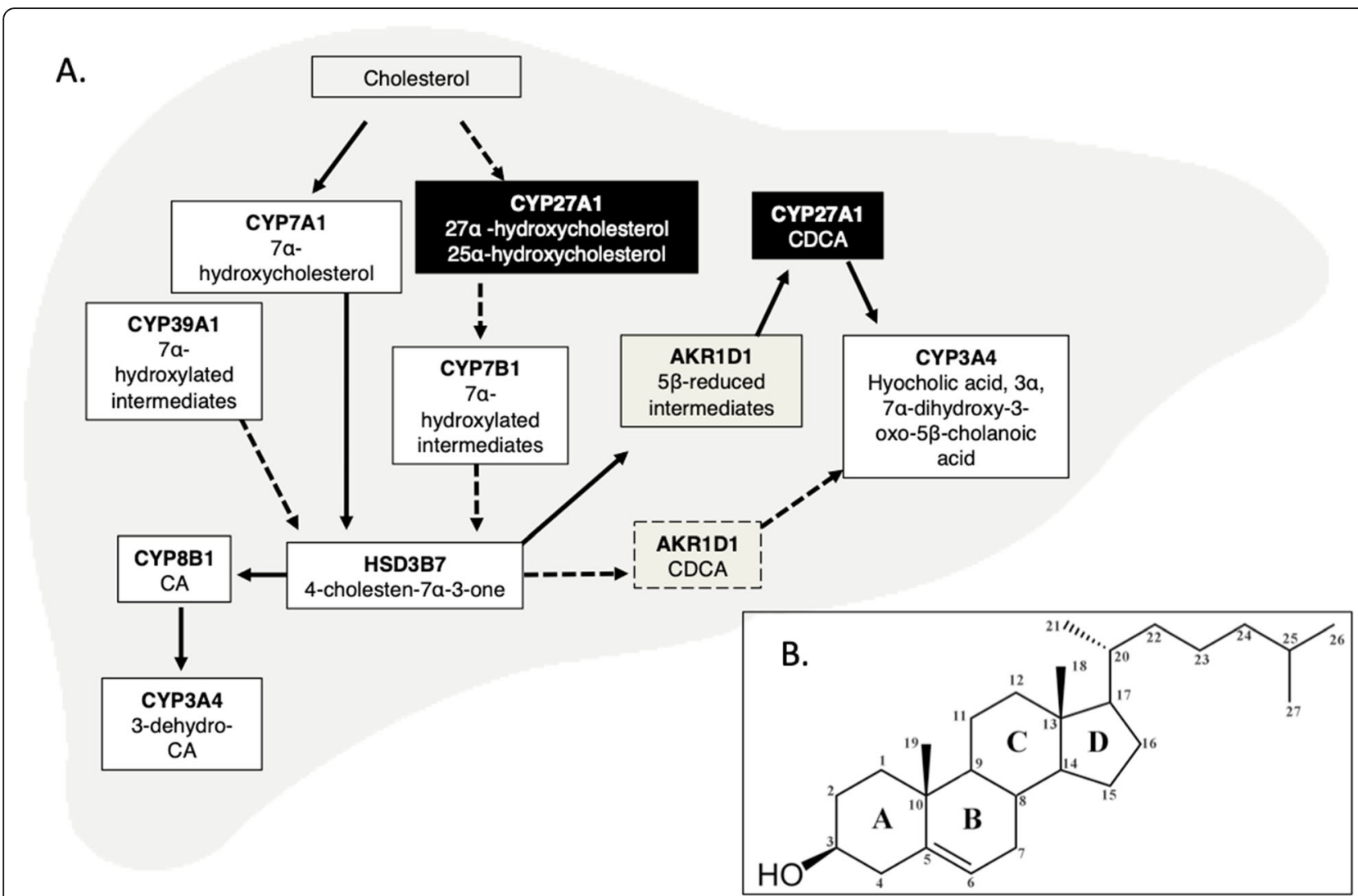

Fig. 1 Schematic overview of bile acid synthesis from cholesterol in the liver. Enzymes in white boxes indicate endoplasmic reticulum localization, while gray boxes indicate cytosolic localization. Black boxes indicate mitochondrial membrane localization. Products of enzymatic reactions are listed below each cytochrome P450. Classical pathway enzymes are linked with solid arrows, and alternative pathway enzymes are connected with dashed arrows [16]. Inset: structure of cholesterol with numbered carbons. CA cholic acid, CDCA chenodeoxycholic acid. Adapted from Fuchs, 2003

restoring appropriate systemic cholesterol concentrations [27]. In agreement with these data, Cyp7a1 knockout mice are resistant to developing metabolic disorders and have increased glucose sensitivity in response to high dietary fat and cholesterol [15].

As in mice, humans with mutations in Cyp $7 a 1$ that result in loss of function exhibit significantly reduced BA pool as low as $6 \%$ of the normal size [28]. Cyp7a1 mutations correlate with high levels of LDL cholesterol and elevated hepatic cholesterol that can lead to premature atherosclerosis [28]. Supplementation with CA restores BA pool size, BA and sterol excretion, and fractional cholesterol absorption, but also increases intestinal and hepatic cholesterol levels [15, 29]. Additionally, Cyp7a1 knockout mice expressing human Cyp7a1 exhibit different responses to altered diets than wild-type counterparts [17]. This discrepancy is likely due to the lack of an LXR $\alpha$ binding sequence in the promoter region of the human gene [17]. BA composition can, therefore, be experimentally manipulated highlighting the potential usefulness of CYP7A1 as a therapeutic target for human disease.
Indeed, transgenic mice overexpressing Cyp $7 a 1$ are resistant to obesity, fatty liver disease, and insulin resistance when fed a high-fat diet and exhibit both increased secretion of very low-density lipoproteins (VLDL) and a more hydrophobic BA pool [30].

\section{CYP7A1 in males versus females}

Male Cyp7a1 knockout mice have lower levels of CA, smaller BA pools, and lower rates of intestinal cholesterol absorption than females despite the lack of BA synthesis via the classical pathway [27, 30,31]. Male null mice also demonstrate a negligible response to increased dietary cholesterol. However, females exhibited a threefold increase in hepatic cholesterol levels and a lithogenic gallbladder composition [31, 32]. These data suggest that differences in alternative BA pathway activity in Cyp7a1deficient mice involve sexually dimorphic regulators other than cholesterol [31]. Indeed, recent evidence suggests a sexual dimorphism in the regulation of Cyp7a1 by thyroid hormone (T3), where $\mathrm{T} 3$ can reduce Cyp7a1 mRNA and protein in male but not female mice that express human 
CYP7A1 [33]. However, this difference does not seem to extend to humans with thyroid dysfunction [33] (Table 2). Interestingly, maternal diet also affects murine hepatic function in a sexually dimorphic manner; male offspring from mothers who consumed high-fat diets had reduced expression of Cyp7a1 with concomitant lipid storage in the liver. Male offspring also exhibited increased Cyp $8 b 1$ expression, similar to the Cyp7a1 knockout mice [34].

\section{CYP8B1}

12- $\alpha$-Hydroxylase (CYP8B1) catalyzes 12- $\alpha$-hydroxylation of substrates emerging from both the classical and alternative pathways [35] (Fig. 1). Substrates are either converted by CYP8B1 to precursors of CA, or by aldo-keto redutase 1D1 (AKR1D1) to precursors of CDCA [8]. While CYP7A1 controls the size of the BA pool produced, activity of CYP8B1 controls the ratio of CA to CDCA in the BA pool; decreased activity results in increased CDCA and a more hydrophobic BA pool, whereas increased activity results in increased $\mathrm{CA}$ and a more hydrophilic BA pool [36, 37]. Cholesterol uptake, phospholipid transport in the liver, and hepatotoxicity are also regulated by differential expression of CYP8B1 [36, 37]. CA is an established ligand for FXR and association with FXR induces expression of $S H P$, a negative regulator of both Cyp $8 b 1$ and Cyp7a1 transcription [19]. In the presence of BAs, hepatic nuclear factor 4 alpha (HNF4 $\alpha$ ) downregulates Cyp $8 b 1$ transcription via upregulation of Shp [37]. Peroxisome proliferator activated receptor alpha (PPAR $\alpha)$ is also a regulator of both Cyp7a1 and Cyp8b1, and signaling from retinoic acid-related orphan receptor- $\alpha$ may additionally have a role in Cyp8b1 expression [38, 39]

In Cyp $8 b 1$ knockout mice, the amount of CA produced is significantly reduced [40]. In response, the classical pathway enzyme CYP7A1 is upregulated, due to a lack of negative regulation, to produce more CDCA that compensates for missing CA; the size of the BA pool is not only restored, but is significantly increased in both male and female mice [3, 40]. Knockout of Cyp8b1 ultimately causes steatorrhea and related symptoms attributed to decreased intestinal absorption of lipids and BA reuptake [35, 40]. However, targeted inhibition protects against development of type 2 diabetes mellitus and cholestasis [35, 40].

Upregulation of Cyp $8 b 1$ when its negative inhibitor SHP is genetically deleted decreases deposition of atherosclerotic plaques when accompanied by apolipoprotien $\mathrm{E}$ (APOE) knockout in spite of dietary CA supplementation or high fat $[41,42]$. Conversely, genetic removal of both Cyp $8 b 1$ and APOE increased aortic plaques [43]. Although other regulators have been identified, this apparent susceptibility to the SHP/FXR pathway and reversal by CA makes the gene potentially well suited for targeted pharmacological upregulation [42].

\section{Cyp8b1 in males and females}

In mice lacking expression of Cyp8b1, the total BA pool increases in males by a larger amount than females: $37 \%$ in males and 20\% in females [3] (Table 2). Moreover, female mice lacking Cyp8b1 expression also have significantly more CA secondary to higher CYP7A1 activity [3]. As a result of higher BA production in knockout and wild-type females, greater intestinal absorption of sterols is observed compared to males [3]. In fasted wild-type female rabbits, a sevenfold increase in mRNA levels and enzymatic activity was observed; however, in fasted male rabbits, no alteration of either mRNA or activity of CYP8B1 was observed [44, 45].

\section{CYP27A1}

Sterol 27-hydroxylase (CYP27A1) primarily hydroxylates cholesterol to 27-hydroxycholesterol in the first step of BA synthesis in the alternative pathway [46] (Fig. 1). The enzyme also hydroxylates $\mathrm{C}-27$ intermediates produced by CYP7A1 in the classical pathways [47]. Expression and activity of CYP27A1 can be regulated transcriptionally by altering the stability of its mRNA and through variation of available substrate $[48,49]$. Specificity protein 1 (SP-1) and HNF4 $\alpha$ binding sites and a BARE in the promoter region of the gene have each been reported in human and rat, which when exposed to BAs, produces a downregulation of Cyp27a1 mRNAs [50,51]. Like other enzymes in the BA synthesis pathways, upregulation of Cyp27a1 and BA synthesis is induced by cholesterol [52]. Indeed, overexpression of Cyp27a1 is sufficient to increase BA synthesis, suggesting a role for the enzyme in responding to hyperlipidemia $[52,53]$. In vitro experiments in human hepatocytes have also revealed a role for growth hormone, insulin-like growth factor-1, and glucocorticoids in upregulating activity of CYP27A1 [54]. Further research is necessary to determine the clinical relevance of these data.

More than 30 different mutations in Cyp27a1 cause cerebrotendinous xanthomatosis (CTX) in humans, which is associated with a variety of symptoms including abnormal synthesis of BAs and deposition of cholesterol and its derivatives primarily in the nervous system and tendons [55]. Patients with CTX lack appropriate regulation of Cyp7a1, leading to an accumulation of cholestanol and C-27 bile alcohols [56]. This phenotype manifests as premature, rapidly progressing atherosclerosis and coronary artery disease [57]. However, the symptoms of CTX are not exclusively related to deficiencies in liver BA synthesis, suggesting extrahepatic roles for CYP27A1 or its products in humans. Indeed, Cyp27a1 is expressed in many extrahepatic tissues, likely due to its presence in macrophages and endothelial cells, where it plays an important role in the hydroxylation of the C-27 vitamin $\mathrm{D}_{3}$ [58]. Notably, genetic removal of Cyp27a1 in mice does not fully recapitulate the symptoms 
of CTX, revealing an important species divergence in the function or localization of the enzyme [59]. Cyp27a1 knockout mice exhibit reduced BA synthesis, increased expression of Cyp7a1, and elevated serum lipid profiles, similar to patients with CTX $[60,61]$. However, none of the tendon or neurological phenotypes are present in the Cyp27a1 knockout mice [61].

\section{CYP27A1 in males and females}

Interest in sexually dimorphic drug metabolism and liver disease development lead to several studies describing Cyp27a1 expression and activity differences in adult males and females. Basal levels of Cyp27a1 expression appear to be equal in men and women; however, the concentration of its product, 27-hydroxycholesterol, is lower in females suggesting differences in activity levels [62]. The presence of high cholesterol in the diet causes a downregulation of Cyp27a1 expression in males and females equally [63] (Table 2). The sex of knockout mice was not indicated in previous studies.

\section{AKR1D1}

Aldo-keto redutases are a conserved group of NADPHdependent oxido-reductase enzymes that reduce ketosteroids [64, 65] (Fig. 1). The 5 $\beta$-reductases (AKR1D1-3) comprise a unique subfamily that catalyzes the reduction of double bonds of $\Delta 4-3$-ketosterols in an efficient and stereospecific manner based on residues presumably located at their active sites $[66,67]$. Although the subfamily includes three isoforms, only AKR1D1 is expressed in humans [68].

High concentrations of CDCA are toxic to hepatocytes, and AKR1D1 enzymatic activity is a key regulatory point in controlling the balance of BAs [69]. For example, overexpression of AKR1D1 in isolated human hepatocytes leads to increased expression of CYP3A4 and other CYPs involved in metabolism of xenobiotics. Conversely, genetic reduction of akrld1 reduces expression of cytochrome P450s, similar to diabetic patients in whom decreased hepatic expression of $A k r l d 1$ and decreased production of CDCA are observed [70]. CDCA decreases plasma lipids in hypertriglyceridemic patients; the mechanisms mediating this effect in diabetic patients are currently unknown [71]. Additionally, infants with a deficiency in 5 3 reductase activity have reduced primary BA synthesis and accumulation of $\Delta 4$-3-keto- and $5 \alpha$-reduced (allo-) BAs [66]. Effects of this metabolic disorder are severe and manifest as cholestasis and neonatal liver damage, which are likely caused by the accumulation of potentially hepatotoxic levels of BAs [66].

\section{AKR1D1 in males and females}

Male mice with genetic removal of $A k r 1 d 1$ exhibit fourfold higher BA concentrations in the liver and lower body fat compared to females [72]. Akr1d1-deficient mice also display a sexually dimorphic metabolic phenotype with female mice being protected from the adverse metabolic effects of a high-fat diet. In mature mice lacking ark1d1, no differences in glucose tolerance is observed, and mice are the same weight as wild-type counterparts [73]. However, after 20 weeks of high-fat diet feeding, female Akr1d1 knockout mice are protected from diet-induced weight gain, unlike males, who have enhanced insulin sensitivity, suggesting a role in metabolic diseases [73].

\section{CYP3A4}

Members of the CYP3A family are the most abundant CYP450s in the liver and are responsible for the metabolism of approximately $50 \%$ of pharmaceuticals available in the USA [74, 75]. Among the four CYP3A isoforms in humans, CYP3A4 is the most highly expressed; eight CYP3A isoforms are expressed in mice [59]. In addition to its role in drug metabolism, CYP3A4 converts cholesterol to $4 \beta$-hydroxycholesterol and regulates lipid metabolism as an activator of the LXR $\alpha$ receptor [75, 76] (Fig. 1). CYP3A4 is also responsible for protecting the liver against the toxic effects of high concentrations of BAs thereby serving as a master regulator of expression of many enzymes involved in BA synthesis to protect against cholestasis [77].

Expression of Cyp3a causes an accumulation of 25hydroxycholesterol, while genetic deletion of the enzyme significantly reduces the concentration [78]. Not only is 25-hydroxycholesterol a precursor to BAs, but it is also an oxysterol that suppresses the sterol sensor SREBP-2 and downregulates de novo cholesterol synthesis [78]. Lower cholesterol levels were observed in livers of Cyp3a knockout mice; however, more studies are required to clarify the role of CYP3A4 in cholesterol homeostasis [78]. Differences in CYP3A4 expression between individuals can be greater than 50 -fold. While over 30 single-nucleotide polymorphisms have been identified, they occur at a frequency of less than $5 \%$ in humans and are consistently heterozygous, suggesting that individual differences may result from other mechanisms $[79,80]$.

Post-translational modifications are predicted to significantly regulate CYP3A4 activity and expression [80]. The protein has at least three phosphorylation sites, although phosphorylation may be related to ubiquitination [81]. Two miRNAs have also been identified to regulate CYP3A4 [80]. One inhibits expression in human embryonic kidney 293 cells, and the other negatively regulates human pregnane $\mathrm{X}$ receptor (PXR) and therefore, indirectly inhibits CYP3A4 translation [80]. In silico methods have identified additional miRNAs that may significantly regulate expression, though more research is needed [80].

\section{CYP3A4 in males and females}

Significant sex differences attributed to CYP3A4 activity have been described in the context of drug and 
xenobiotic metabolism. Expression and activity of CYP3A4 are higher in women compared to men, a difference that is diminished after menopause with the loss of estrogen [82]. Differences in rates of cholestasis in women, especially caused by pregnancy, are thought to be attributed to differential regulation of CYP3A4 [83]. However, interpretation of data should be made with caution due to the important role of CYP3A4 in the metabolism of estrogen [84].

\section{The influence of biological sex on BA synthesis}

Biological sex has long been recognized as an important modulator of cardiovascular and hepatic diseases [85-88]. Although differences in body composition, hormonal status, and fat distribution complicate interpretation of data, it is clear that premenopausal women compared to agematched men are at lower risk of developing non-toxinrelated liver and cardiovascular diseases including those attributable to elevated serum cholesterol [89, 90]. The lipid profiles of pre-menopausal women are less pro-atherogenic with higher concentrations of high-density lipoproteins containing cholesterol [91]. Sexual dimorphisms in serum cholesterol extend to the liver where sex differences are also observed in BA pool composition and size [92, 93].

The release pattern of growth hormone and subsequent control of signal transducer and activator of transcription $5 b$ (STAT5b) are involved in sexual dimorphism of hepatic CYPs [94]. Other hepatic transcription factors involved in sex-specific expression of $\mathrm{P} 450 \mathrm{~s}$ include hepatocyte nuclear factor $4 \alpha$ (HNF4 $\alpha$ ) [69] and retinoid X receptor $(\mathrm{RXR} \alpha)$, the co-receptor for many nuclear receptors in hepatocytes [95]. When HNF4 $\alpha$ expression is removed, for example, 372 sex-specific genes are specifically affected in the livers of male mice versus only 61 in the females. Additionally, in female mice, the BA pool is approximately $60 \%$ larger and more hydrophobic than in males, and higher fecal levels of excreted BAs are also observed in females [91, 93]. The composition of the bile acid pool is also sexually dimorphic with females producing more CDCA than males [96]. The excess BAs cannot be attributed to Cyp7a1 expression, which is paradoxically lower in females [4]. However, when challenged with a high-cholesterol diet, female mice exert a 50\% higher rate of CYP7A1 activity compared to males [4]. These data significantly confound interpretation of data from rodent models: unlike female mice, women have lower BA pool size compared to men [92] (Table 2).

\section{The role of sex hormones in cholesterol homeostasis}

Female sex and estrogens are emerging as important regulators of BA production and through critical hepatic feedback mechanisms, serum cholesterol levels. Most of the early data on sex differences in serum lipid profiles, BA synthesis, and BA pool composition were derived from observations of pre- versus postmenopausal women, of individuals receiving estrogen supplementation, and of women with polycystic ovarian syndrome in whom circulating sex hormone levels are abnormal [97, 98]. Nearly half of women administered an estrogen receptor (ER) antagonist as a treatment for certain breast cancers develop hepatic steatosis within 2 years of beginning treatment [99]. Similarly, mice that are deficient in estrogen or are not responsive to estrogen signaling are obese and have elevated triglycerides levels that are linked to the development of hepatic steatosis, a condition that is reversible through administration of estrogen [100, 101]. Steatosis that develops in the setting of depleted estrogen may be further exacerbated by exposure to endocrine disruptors with possible estrogen-blocking effects like bisphenol A present in many plastics [102]. Interestingly, in women, high levels of circulating estrogen during pregnancy are associated with development of cholestasis via a mechanism that likely involves inhibition of BA transport to the liver from the intestines rather than synthesis $[103,104]$. These conflicting effects may also be explained by the use of both physiological and non-physiological concentrations of estrogen. Despite this, both synthetic and endogenous estrogens have been implicated in sex differences observed in liver dysfunction and are thought to be generally beneficial at normal levels in premenopausal women in terms of preventing and limiting progression of liver and cardiac diseases $[105,106]$.

\section{Transcriptional effects of estrogen}

Estrogen exerts genomic effects through ligand-bound ERs that translocate to the nucleus and bind to estrogen response elements (EREs). Non-genomic effects are also mediated by membrane-bound ERs via activation of cell signaling cascades [107]. Although the impacts are best described in breast cancer cells, the significance of the modulatory effects of estrogen in the liver is being explored. Estrogen-induced cholestasis is caused by reduced BA synthesis and transport [108]. Hepatocytes express ER $\alpha$ and are, therefore, responsive to both the genomic and non-genomic effects of estrogen [109]. Rat hepatocytes exposed to physiological levels of estrogen exhibit increased CYP7A1 activity along with small transient increases in BA production [110]. However, in vivo effects appear to be diet- and time-dependent. Similarly, livers of ovariectomized baboons on a high fat and cholesterol diet exhibited higher activity of CYP7A1 [111]. Single injection of supraphysiological concentrations of estrogen in rats did not induce changes in CYP7A1 activity at the level of the microsome, whereas 21-day treatment inhibited activity [112]. It is, therefore, unclear whether cholestasis caused by various estrogen supplementation therapies is due solely to altered CYP7A1 
expression or activity [113, 114]. Through activation of the estrogen receptor ER- $\alpha$, synthetic estrogen also upregulates Cyp $7 b 1$ and decreases CYP8B1 signaling; ER- $\beta$ does not appear to have a role in regulation of BA synthesis enzymes. A recently identified but not well understood estrogen receptor, GPR30, also appears to positively regulate expression of Cyp7a1 [115] (Table 3).

An ERE has been identified in the promoter region of Akr1d1 [64]. Higher levels of estrogen may help to reduce the effects of AKR1D1-deficient individuals, since testosterone is an inhibitory substrate for AKR1D1 [116]. Testosterone has two binding sites that both block binding of other hormones like estrogen to AKR1D1 [116]. EREs have not been identified in many cytochrome P450s involved in BA synthesis; however, other proteins and sequences like the transcription factors activator protein 1 (AP-1) and Sp-1 and half-palindromic estrogen response sequences (half-sites) in promoters can mediate the transcriptional activity of nuclear ERs. Estrogen interacts with $\mathrm{Sp}-1$, and this association is required for enhanced transcription of many genes including $R X R \alpha$ and $L X R \alpha$ [117]. In agreement with this, expression of $R X R \alpha$ is significantly higher in the livers of females compared to males [118]. Additionally, CYP3A4 has an important role in the 4-hydroxylation of estrogen, the first step in hepatic metabolism of estrogens [84]. Accordingly, estrogen itself negatively regulates expression of Cyp3a4, likely through interactions with ERs in the promoter region of the gene $[119,120]$. Indeed, as age increases and estrogen levels decline in women, hepatic levels of CYP3A4 are reduced [119].

Estrogen exerts effects on BA synthesis that influence enzymatic activity as well as BA pool composition. Cyp27a1 expression, for example, is inhibited in HepG2 liver-derived cells by estrogen treatment. Both ER $\alpha$ and ER $\beta$ associate with the promoter region of the Cyp27a1 gene to inhibit expression; conversely, androgens promote expression of Cyp27a1 in the same cell line likely via induction of JNK signaling [121, 122]. Interestingly, CYP27A1 enhances ER-ERE interactions in liver cells and may promote upregulation of Cyp7b1 [123]. Transfection of human embryonic kidney 293 cells with ER $\alpha$ and ER $\beta$ combined with estrogen treatment upregulates expression and activity of CYP7B1 [124]. Cyp $7 b 1$ expression is negatively regulated by androgens in prostate cancer cells, in which opposing effects of estrogen are observed [124]. In agreement with these data, examination of the composition of the BA pool has revealed that men have higher CDCA than women [6]. Additionally, in animal models, the concentration of CDCA in bile is reduced with estrogen signaling through $\mathrm{ER} \alpha[125,126]$.

\section{Conclusions}

Drug development for atherosclerosis and BA synthesis deficiencies are increasingly focused on BA biosynthetic pathways. Potential pharmacological targets include the nuclear receptors FXR and SHP; however, excretion of BAs to prevent cytotoxic concentrations will need to be considered. For example, although SHP itself lacks a DNA-binding domain, it interacts with multiple nuclear receptors including ERs, thereby inhibiting their transcription $[127,128]$. The SHP promoter harbors an AP-1 binding site that when mutated, removes negative regulation induced by BAs [129]. The biological effects of estrogen on these nuclear receptors appear to vary based on cell type; therefore, it is critical to thoroughly examine their effects in hepatocytes and in the liver in vivo.

Sex differences in the therapeutic response to compounds that target BA synthesis may vary significantly not only with respect to the CYPs that mediate drug metabolism but also in the CYPs that regulate BA concentration and composition between the sexes. Lessons from cardiovascular disease should inform the development of these therapies, and a complete understanding of molecular sexual dimorphisms regulating BA synthesis will help in addressing these issues. Importantly, post-menopausal women may be less sensitive to drugs that intend to increase BA synthesis due to diminished levels of estrogen that normally promote activity of enzymes that produce BAs. Whole-genome examination of promoters for canonical ERE $s$ has been performed, revealing no perfect or near-perfect estrogen binding sites for enzymes involved in BA synthesis except AKR1D1 [130]. However, half-ERE sites that bind SP-1 and AP-1 sites should be considered in greater detail to understand the role of estrogen in regulation in BA synthesis and cholesterol homeostasis in the liver.

\section{Perspectives and significance}

Cholesterol homeostasis has been recognized as an important modulator of the cardiovascular system in health and disease. Indeed, drugs that lower systemic cholesterol such as statins that reduce hepatic cholesterol production improve outcomes of cardiovascular diseases. However, neglecting to consider sex differences in the expression and activity of lipid-handling proteins targeted by cholesterol-lowering drugs has led to limitations in their utility. For example, women experience a significantly higher incidence of myalgia and reduced survival benefit from statins compared to men (Legato et al., [131]). Strategies that reduce cholesterol through modulation of BA synthesis may benefit those who cannot tolerate statins or for whom the drugs are ineffective. To avoid unexpected effects due to biological sex, development of these drugs should address sex differences in the enzymes that produce BAs.

Sex differences in BA synthesis have been reported in humans and rodents for nearly 50 years. Lessons from studies on cholesterol in cardiac health should inform 
further examination of the roles of both estrogen and androgens in regulating expression of enzymes involved in BA synthesis. We recommend systematic experiments that include addition of exogenous estrogen and androgens and gonadectomized males. This system would allow examination of the roles of both androgens and estrogen. The resulting phenotypes could reveal important information about not only mechanisms regulating BA production but also about the possible hepatic effects of gender-affirming hormone supplementation in transgender individuals, a vastly underrepresented field of study. Comparable studies performed in mice that examine cardiac function have found detrimental effects in gonadectomized mice receiving doses of estrogen relevant to the serum of pre-menopausal females. The same may be true for BA synthesis and cholesterol homeostasis because similar mechanisms mediate the regulation of genes important to bile acid synthesis.

\begin{abstract}
Abbreviations
AKR1D1: Aldo-keto reductase 1D1; AP-1: Activator protein 1; APOE: Apolipoprotein E; BA: Bile acid; BARE: Bile acid response element: CA: Cholic acid; CDCA: Chenodeoxycholic acid; CTX: Cerebrotendinous xanthomatosis; CYP: Cytochrome P450; CYP7A1: Cholesterol 7a-hydroxylase; CYP7B1: Oxysterol 7a-hydroxylase; CYP8B1: 12-Alpha-Hydroxylase; CYP27A1: Sterol 27-hydroxylase; ER: Estrogen receptor; ERE: Estrogen response element; FGF: Fibroblast growth factor; FXR: Farnesoid X receptor; HNF4a: Hepatic nuclear factor 4 alpha; JNK: c-jun N-terminal kinase: LRH1: Liver receptor homolog 1; LXRa: Liver X receptor alpha; PXR: Pregnane $X$ receptor; RXRa: Retinoid X receptor alpha; SHP: Small heterodimer partner; SP-1: Specificity protein 1; SREBP: Sterol regulatory element-binding protein; STAT: Signal transducer and activator of transcription
\end{abstract}

\section{Acknowledgements}

The authors acknowledge Ann Li for her contributions to early versions of this manuscript.

\section{Authors' contributions \\ Each author (TP, ES, ER, HC, and PH) contributed to the design and production of the manuscript. TP coordinated edits. ES and ER made substantial editorial contributions. PH and TP conceived the manuscript, and $\mathrm{PH}$ provided expertise in the oversight in the development of the text. Each author has read and approved of the submitted version of this manuscript and takes responsibility for the content presented.}

\section{Funding}

This work was supported by University of Colorado Boulder Arts \& Sciences Support of Education Through Technology faculty fellowship (P.A.H.) and Howard Hughes Medical Institute Undergraduate Research Opportunity Program faculty and team grants (P.A.H.). Publication of this article was funded by the University of Colorado Boulder Libraries Open Access Fund.

\section{Availability of data and materials}

Not applicable.

\section{Ethics approval and consent to participate}

Not applicable.

\section{Consent for publication}

Not applicable.

\section{Competing interests}

The authors declare that they have no competing interests.
Received: 27 May 2019 Accepted: 11 September 2019

Published online: 27 November 2019

\section{References}

1. Lorbek G, Lewinska M, Rozman D. Cytochrome P450s in the synthesis of cholesterol and bile acids--from mouse models to human diseases. FEBS J. 2012;279(9):1516-33

2. Chiang JY. Bile acid metabolism and signaling. Compr Physiol. 2013;3(3):1191-212

3. Li-Hawkins J, Gafvels M, Olin M, Lund EG, Andersson U, Schuster G, et al. Cholic acid mediates negative feedback regulation of bile acid synthesis in mice. J Clin Invest. 2002;110(8):1191-200.

4. Turley SD, Schwarz M, Spady DK, Dietschy JM. Gender-related differences in bile acid and sterol metabolism in outbred CD-1 mice fed low- and highcholesterol diets. Hepatology. 1998;28(4):1088-94.

5. Xiang X, Backman JT, Neuvonen PJ, Niemi M. Gender, but not CYP7A1 or SLCO1B1 polymorphism, affects the fasting plasma concentrations of bile acids in human beings. Basic Clin Pharmacol Toxicol. 2012;110(3):245-52.

6. Frommherz L, Bub A, Hummel E, Rist MJ, Roth A, Watzl B, et al. Age-related changes of plasma bile acid concentrations in healthy adults-results from the cross-sectional KarMeN study. PLoS One. 2016;1 1(4):e0153959.

7. Chiang JY. Bile acids: regulation of synthesis. J Lipid Res. 2009;50(10):1955-66.

8. Thomas C, Pellicciari R, Pruzanski M, Auwerx J, Schoonjans K. Targeting bile-acid signalling for metabolic diseases. Nat Rev Drug Discov. 2008; 7(8):678-93

9. Li T, Apte U. Bile acid metabolism and signaling in cholestasis, inflammation, and cancer. Adv Pharmacol. 2015;74:263-302

10. Monte MJ, Marin JJ, Antelo A, Vazquez-Tato J. Bile acids: chemistry, physiology, and pathophysiology. World J Gastroenterol. 2009;15(7):804-16.

11. Axelson M, Sjovall J. Potential bile acid precursors in plasma-possible indicators of biosynthetic pathways to cholic and chenodeoxycholic acids in man. J Steroid Biochem. 1990;36(6):631-40.

12. Clinical Biochemistry of Domestic Animals. 6th ed. Kaneko J, Harvey J, Bruss M, editors. Burlington, MA: Elsevier, Inc.; 2008.

13. Bonde $Y$, Eggertsen $G$, Rudling M. Mice Abundant in muricholic bile acids show resistance to dietary induced steatosis, weight gain, and to impaired glucose metabolism. PLoS One. 2016;11(1):e0147772.

14. Miller WL, Auchus RJ. The molecular biology, biochemistry, and physiology of human steroidogenesis and its disorders. Endocr Rev. 2011;32(1):81-151.

15. Ferrell JM, Boehme S, Li F, Chiang JY. Cholesterol 7alpha-hydroxylasedeficient mice are protected from high-fat/high-cholesterol diet-induced metabolic disorders. J Lipid Res. 2016:57(7):1144-54.

16. Fuchs M. Bile acid regulation of hepatic physiology. III. Regulation of bile acid synthesis: past progress and future challenges. Am J Physiol Gastrointest Liver Physiol. 2003;284(4):G551-7.

17. Chen JY, Levy-Wilson B, Goodart S, Cooper AD. Mice expressing the human CYP7A1 gene in the mouse CYP7A1 knock-out background lack induction of CYP7A1 expression by cholesterol feeding and have increased hypercholesterolemia when fed a high fat diet. J Biol Chem. 2002;277(45):42588-95.

18. Inagaki T, Choi M, Moschetta A, Peng L, Cummins CL, McDonald JG, et al. Fibroblast growth factor 15 functions as an enterohepatic signal to regulate bile acid homeostasis. Cell Metab. 2005;2(4):217-25.

19. Goodwin B, Jones SA, Price RR, Watson MA, McKee DD, Moore LB, et al. A regulatory cascade of the nuclear receptors FXR, SHP-1, and LRH-1 represses bile acid biosynthesis. Mol Cell. 2000;6(3):517-26.

20. Kong B, Wang L, Chiang JY, Zhang Y, Klaassen CD, Guo GL. Mechanism of tissue-specific farnesoid $X$ receptor in suppressing the expression of genes in bile-acid synthesis in mice. Hepatology. 2012;56(3):1034-43.

21. Kim I, Ahn SH, Inagaki T, Choi M, Ito S, Guo GL, et al. Differential regulation of bile acid homeostasis by the farnesoid $\mathrm{X}$ receptor in liver and intestine. $J$ Lipid Res. 2007;48(12):2664-72.

22. Hofmann AF. Bile Acids: The Good, the Bad, and the Ugly. News Physiol Sci. 1999:14:24-9.

23. Cohen BI, Raicht RF, Mosbach EH. Sterol metabolism studies in the rat. Effects of dietary plant sterols and bile acids on sterol metabolism. Biochim Biophys Acta. 1977;487(2):287-96.

24. Reynier MO, Montet JC, Gerolami A, Marteau C, Crotte C, Montet AM, et al. Comparative effects of cholic, chenodeoxycholic, and ursodeoxycholic acids on micellar solubilization and intestinal absorption of cholesterol. J Lipid Res. 1981;22(3):467-73. 
25. Powell AA, LaRue JM, Batta AK, Martinez JD. Bile acid hydrophobicity is correlated with induction of apoptosis and/or growth arrest in HCT116 cells. Biochem J. 2001;356(Pt 2):481-486.

26. Wang $H$, Chen J, Hollister $\mathrm{K}$, Sowers LC, Forman BM. Endogenous bile acids are ligands for the nuclear receptor FXR/BAR. Mol Cell. 1999;3(5):543-53.

27. Jones RD, Lopez AM, Tong EY, Posey KS, Chuang JC, Repa JJ, et al. Impact of physiological levels of chenodeoxycholic acid supplementation on intestinal and hepatic bile acid and cholesterol metabolism in Cyp7a1deficient mice. Steroids. 2015;93:87-95.

28. Pullinger CR, Eng C, Salen G, Shefer S, Batta AK, Erickson SK, et al. Human cholesterol 7alpha-hydroxylase (CYP7A1) deficiency has a hypercholesterolemic phenotype. J Clin Invest. 2002;110(1):109-17.

29. Jones RD, Repa JJ, Russell DW, Dietschy JM, Turley SD. Delineation of biochemical, molecular, and physiological changes accompanying bile acid pool size restoration in Cyp7a1(-/-) mice fed low levels of cholic acid. Am J Physiol Gastrointest Liver Physiol. 2012;303(2):G263-74.

30. Li T, Owsley E, Matozel M, Hsu P, Novak CM, Chiang JY. Transgenic expression of cholesterol 7alpha-hydroxylase in the liver prevents high-fat diet-induced obesity and insulin resistance in mice. Hepatology. 2010;52(2):678-90.

31. Schwarz M, Russell DW, Dietschy JM, Turley SD. Alternate pathways of bile acid synthesis in the cholesterol 7alpha-hydroxylase knockout mouse are not upregulated by either cholesterol or cholestyramine feeding. J Lipid Res. 2001;42(10):1594-603.

32. Erickson SK, Lear SR, Deane S, Dubrac S, Huling SL, Nguyen L, et al. Hypercholesterolemia and changes in lipid and bile acid metabolism in male and female cyp7A1-deficient mice. J Lipid Res. 2003;44(5):1001-9.

33. Drover VA, Agellon LB. Regulation of the human cholesterol 7alphahydroxylase gene (CYP7A1) by thyroid hormone in transgenic mice. Endocrinology. 2004;145(2):574-81.

34. Wankhade UD, Zhong Y, Kang P, Alfaro M, Chintapalli SV, Piccolo BD, et al. Maternal High-Fat Diet Programs Offspring Liver Steatosis in a Sexually Dimorphic Manner in Association with Changes in Gut Microbial Ecology in Mice. Sci Rep. 2018;8(1):16502.

35. Kaur A, Patankar JV, de Haan W, Ruddle P, Wijesekara N, Groen AK, et al. Loss of Cyp8b1 improves glucose homeostasis by increasing GLP-1. Diabetes. 2015;64(4):1168-79.

36. Li T, Chiang JY. Bile acid signaling in metabolic disease and drug therapy. Pharmacol Rev. 2014;66(4):948-83.

37. Zhang $\mathrm{M}$, Chiang JY. Transcriptional regulation of the human sterol 12alpha-hydroxylase gene (CYP8B1): roles of heaptocyte nuclear factor 4alpha in mediating bile acid repression. J Biol Chem. 2001;276(45):41690-9.

38. Mork LM, Strom SC, Mode A, Ellis EC. Addition of dexamethasone alters the bile acid composition by inducing CYP8B1 in primary cultures of human hepatocytes. J Clin Exp Hepatol. 2016;6(2):87-93.

39. Tometsuka C, Koyama YI, Ishijima T, Toyoda T, Teranishi M, Takehana K, et al. Collagen peptide ingestion alters lipid metabolism-related gene expression and the unfolded protein response in mouse liver. Br J Nutr. 2017;117(1):1-11.

40. Murphy C, Parini P, Wang J, Bjorkhem I, Eggertsen G, Gafvels M. Cholic acid as key regulator of cholesterol synthesis, intestinal absorption and hepatic storage in mice. Biochim Biophys Acta. 2005;1735(3):167-75.

41. Kim MJ, Lee KJ, Hwang JY, Lee HS, Chio SH, Lim S, et al. Loss of small heterodimer partner protects against atherosclerosis in apolipoprotein Edeficient mice. Endocr J. 2013;60(10):1171-7.

42. Wang L, Han Y, Kim CS, Lee YK, Moore DD. Resistance of SHP-null mice to bile acid-induced liver damage. J Biol Chem. 2003;278(45):44475-81.

43. Slatis K, Gafvels M, Kannisto K, Ovchinnikova O, Paulsson-Berne G, Parini P, et al. Abolished synthesis of cholic acid reduces atherosclerotic development in apolipoprotein E knockout mice. J Lipid Res. 2010;51(11):3289-98.

44. Eggertsen $\mathrm{G}$, Olin M, Andersson U, Ishida H, Kubota S, Hellman U, et al. Molecular cloning and expression of rabbit sterol 12alpha-hydroxylase. J Biol Chem. 1996;271(50):32269-75.

45. Ishida H, Noshiro M, Okuda K, Coon MJ. Purification and characterization of 7 alpha-hydroxy-4-cholesten-3-one 12 alpha-hydroxylase. J Biol Chem. 1992; 267(30):21319-23.

46. Andersson S, Davis DL, Dahlback H, Jornvall H, Russell DW. Cloning, structure, and expression of the mitochondrial cytochrome P-450 sterol 26-hydroxylase, a bile acid biosynthetic enzyme. J Biol Chem. 1989; 264(14):8222-9.

47. Norlin M, von Bahr S, Bjorkhem I, Wikvall K. On the substrate specificity of human CYP27A1: implications for bile acid and cholestanol formation. J Lipid Res. 2003;44(8):1515-22.
48. Chen W, Chiang JY. Regulation of human sterol 27-hydroxylase gene (CYP27A1) by bile acids and hepatocyte nuclear factor 4alpha (HNF4alpha). Gene. 2003;313:71-82.

49. Segev $H$, Honigman A, Rosen $H$, Leitersdorf E. Transcriptional regulation of the human sterol 27-hydroxylase gene (CYP27) and promoter mapping. Atherosclerosis. 2001;156(2):339-47.

50. Twisk J, de Wit EC, Princen HM. Suppression of sterol 27-hydroxylase mRNA and transcriptional activity by bile acids in cultured rat hepatocytes. Biochem J. 1995;305 ( Pt 2):505-511.

51. Ellis E, Axelson M, Abrahamsson A, Eggertsen G, Thorne A, Nowak G, et al. Feedback regulation of bile acid synthesis in primary human hepatocytes: evidence that CDCA is the strongest inhibitor. Hepatology. 2003;38(4):930-8.

52. Pandak WM, Ren S, Marques D, Hall E, Redford K, Mallonee D, et al. Transport of cholesterol into mitochondria is rate-limiting for bile acid synthesis via the alternative pathway in primary rat hepatocytes. J Biol Chem. 2002;277(50):48158-64.

53. Hall E, Hylemon P, Vlahcevic Z, Mallonee D, Valerie K, Avadhani N, et al. Overexpression of CYP27 in hepatic and extrahepatic cells: role in the regulation of cholesterol homeostasis. Am J Physiol Gastrointest Liver Physiol. 2001;281(1):G293-301.

54. Araya Z, Tang W, Wikvall K. Hormonal regulation of the human sterol 27hydroxylase gene CYP27A1. Biochem J. 2003;372(Pt 2):529-534.

55. Gupta RP, Patrick K, Bell NH. Mutational analysis of CYP27A1: assessment of 27-hydroxylation of cholesterol and 25-hydroxylation of vitamin D. Metabolism. 2007;56(9):1248-55.

56. Rosen H, Reshef A, Maeda N, Lippoldt A, Shpizen S, Triger L, et al. Markedly reduced bile acid synthesis but maintained levels of cholesterol and vitamin D metabolites in mice with disrupted sterol 27-hydroxylase gene. J Biol Chem. 1998;273(24):14805-12.

57. Fujiyama J, Kuriyama M, Arima S, Shibata Y, Nagata K, Takenaga S, et al. Atherogenic risk factors in cerebrotendinous xanthomatosis. Clin Chim Acta. 1991;200(1):1-11.

58. Babiker A, Andersson O, Lund E, Xiu RJ, Deeb S, Reshef A, et al. Elimination of cholesterol in macrophages and endothelial cells by the sterol 27hydroxylase mechanism. Comparison with high density lipoproteinmediated reverse cholesterol transport. J Biol Chem. 1997;272(42):26253-61.

59. Nelson DR, Zeldin DC, Hoffman SM, Maltais LJ, Wain HM, Nebert DW. Comparison of cytochrome P450 (CYP) genes from the mouse and human genomes, including nomenclature recommendations for genes, pseudogenes and alternative-splice variants. Pharmacogenetics. 2004;14(1):1-18.

60. Cali JJ, Hsieh CL, Francke U, Russell DW. Mutations in the bile acid biosynthetic enzyme sterol 27-hydroxylase underlie cerebrotendinous xanthomatosis. J Biol Chem. 1991;266(12):7779-83.

61. Repa JJ, Lund EG, Horton JD, Leitersdorf E, Russell DW, Dietschy JM, et al. Disruption of the sterol 27-hydroxylase gene in mice results in hepatomegaly and hypertriglyceridemia. Reversal by cholic acid feeding. J Biol Chem. 2000:275(50):39685-92.

62. Nunes VS, Panzoldo NB, Leanca CC, Parra ES, Zago VS, da Silva EJ, et al. Increased 27-hydroxycholesterol plasma level in men with low high density lipoprotein-cholesterol may circumvent their reduced cell cholesterol efflux rate. Clin Chim Acta. 2014;433:169-73.

63. Lorbek G, Perse M, Horvat S, Bjorkhem I, Rozman D. Sex differences in the hepatic cholesterol sensing mechanisms in mice. Molecules. 2013;18(9):11067-85.

64. Chen WD, Zhang Y. Regulation of aldo-keto reductases in human diseases. Front Pharmacol. 2012:3:35.

65. Pratt-Hyatt M, Lickteig AJ, Klaassen CD. Tissue distribution, ontogeny, and chemical induction of aldo-keto reductases in mice. Drug Metab Dispos. 2013;41(8):1480-7.

66. Mindnich R, Drury JE, Penning TM. The effect of disease associated point mutations on 5beta-reductase (AKR1D1) enzyme function. Chem Biol Interact. 2011;191(1-3):250-4

67. Faucher F, Cantin L, Luu-The V, Labrie F, Breton R. The crystal structure of human Delta4-3-ketosteroid 5 beta-reductase defines the functional role of the residues of the catalytic tetrad in the steroid double bond reduction mechanism. Biochemistry. 2008;47(32):8261-70.

68. Barski OA, Tipparaju SM, Bhatnagar A. The aldo-keto reductase superfamily and its role in drug metabolism and detoxification. Drug Metab Rev. 2008 40(4):553-624.

69. Wiwi CA, Gupte M, Waxman DJ. Sexually dimorphic P450 gene expression in liver-specific hepatocyte nuclear factor 4alpha-deficient mice. Mol Endocrinol. 2004;18(8):1975-87. 
70. Chaudhry AS, Thirumaran RK, Yasuda K, Yang X, Fan Y, Strom SC, et al. Genetic variation in aldo-keto reductase 1D1 (AKR1D1) affects the expression and activity of multiple cytochrome P450s. Drug Metab Dispos. 2013:41(8):1538-47.

71. Prawitt J, Caron S, Staels B. Bile acid metabolism and the pathogenesis of type 2 diabetes. Curr Diab Rep. 2011;11(3):160-6.

72. Gathercole L, Klusonova P, Nikolaou N, Hazlehurst J, Moolla A, Dempster N, et al. Gender specific metabolic phenotype in the 5[beta]-reductase knockout mouse. Endocrine Abstracts. 2017:49:EP730.

73. Gathercole L, Chapman M, Larner D, Klusonova P, Penning TM, Odermatt A, et al. Female 5beta-reductase knockout mice are protected from diet induced obesity, insulin resistance, and glucose intolerance. Endocrine Abstracts. 2015;38.

74. de Wildt SN, Kearns GL, Leeder JS, van den Anker JN. Glucuronidation in humans. Pharmacogenetic and developmental aspects. Clin Pharmacokinet. 1999;36(6):439-52

75. Pikuleva IA. Cytochrome P450s and cholesterol homeostasis. Pharmacol Ther. 2006;112(3):761-73.

76. Takeda S, Ishii Y, Mackenzie PI, Nagata K, Yamazoe Y, Oguri K, et al. Modulation of UDP-glucuronosyltransferase 2B7 function by cytochrome P450s in vitro: differential effects of CYP1A2, CYP2C9 and CYP3A4. Biol Pharm Bull. 2005;28(10):2026-7.

77. Bodin K, Lindbom U, Diczfalusy U. Novel pathways of bile acid metabolism involving CYP3A4. Biochim Biophys Acta. 2005;1687(1-3):84-93.

78. Hashimoto M, Kobayashi K, Watanabe M, Kazuki Y, Takehara S, Inaba A, et al. Knockout of mouse Cyp3a gene enhances synthesis of cholesterol and bile acid in the liver. J Lipid Res. 2013;54(8):2060-8.

79. Lamba JK, Lin YS, Schuetz EG, Thummel KE. Genetic contribution to variable human CYP3A-mediated metabolism. Adv Drug Deliv Rev. 2002; 54(10):1271-94.

80. Wei Z, Jiang S, Zhang Y, Wang X, Peng X, Meng C, et al. The effect of microRNAs in the regulation of human CYP3A4: a systematic study using a mathematical model. Sci Rep. 2014;4:4283.

81. Wang Y, Liao M, Hoe N, Acharya P, Deng C, Krutchinsky AN, et al. A role for protein phosphorylation in cytochrome P450 3A4 ubiquitindependent proteasomal degradation. J Biol Chem. 2009;284(9):5671-84.

82. Waxman DJ, Holloway MG. Sex differences in the expression of hepatic drug metabolizing enzymes. Mol Pharmacol. 2009;76(2):215-28.

83. Chen J, Zhao KN, Chen C. The role of CYP3A4 in the biotransformation of bile acids and therapeutic implication for cholestasis. Ann Transl Med. 2014;2(1):7.

84. Tsuchiya Y, Nakajima M, Yokoi T. Cytochrome P450-mediated metabolism of estrogens and its regulation in human. Cancer Lett. 2005;227(2):115-24.

85. Reckelhoff JF, Samson WK. Sex and gender differences in cardiovascular, renal and metabolic diseases. Am J Physiol Regul Integr Comp Physiol. 2015;309(9):R1057-9.

86. Spence JD, Pilote L. Importance of sex and gender in atherosclerosis and cardiovascular disease. Atherosclerosis. 2015;241(1):208-10.

87. Kurt Z, Barrere-Cain R, LaGuardia J, Mehrabian M, Pan C, Hui ST, et al. Tissue-specific pathways and networks underlying sexual dimorphism in non-alcoholic fatty liver disease. Biol Sex Differ. 2018;9(1):46.

88. Buzzetti E, Parikh PM, Gerussi A, Tsochatzis E. Gender differences in liver disease and the drug-dose gender gap. Pharmacol Res. 2017;120:97-108.

89. Lloyd-Jones DM, Larson MG, Beiser A, Levy D. Lifetime risk of developing coronary heart disease. Lancet. 1999;353(9147):89-92.

90. Guy J, Peters MG. Liver disease in women: the influence of gender on epidemiology, natural history, and patient outcomes. Gastroenterol Hepatol (N Y). 2013;9(10):633-9

91. Wang X, Magkos F, Mittendorfer B. Sex differences in lipid and lipoprotein metabolism: it's not just about sex hormones. J Clin Endocrinol Metab. 2011;96(4):885-93.

92. Bennion L, Drobny E, Knowler WC, Ginsberg RL, Garnick MB, Adler RD, et al. Sex differences in the size of bile acid pools. Metabolism. 1978;27(8):961-9.

93. Galman C, Angelin B, Rudling M. Pronounced variation in bile acid synthesis in humans is related to gender, hypertriglyceridaemia and circulating levels of fibroblast growth factor 19. J Intern Med. 2011;270(6):580-8.

94. Park SH, Liu X, Hennighausen L, Davey HW, Waxman DJ. Distinctive roles of STAT5a and STAT5b in sexual dimorphism of hepatic P450 gene expression. Impact of STAT5a gene disruption. J Biol Chem. 1999:274(11):7421-30.

95. Cai Y, Dai T, Ao Y, Konishi T, Chuang KH, Lue Y, et al. Cytochrome P450 genes are differentially expressed in female and male hepatocyte retinoid $X$ receptor alpha-deficient mice. Endocrinology. 2003;144(6):2311-8.
96. Fisher MM, Yousef IM. Sex differences in the bile acid composition of human bile: studies in patients with and without gallstones. Can Med Assoc J. 1973;109(3):190-3.

97. Godsland IF. Effects of postmenopausal hormone replacement therapy on lipid, lipoprotein, and apolipoprotein (a) concentrations: analysis of studies published from 1974-2000. Fertil Steril. 2001;75(5):898-915.

98. Soares GM, Vieira CS, de Paula Martins W, Dos Reis RM, de Sa MF, Ferriani RA. Metabolic and cardiovascular impact of oral contraceptives in polycystic ovary syndrome. Int J Clin Pract. 2009;63(1):160-9.

99. Murata Y, Ogawa Y, Saibara T, Nishioka A, Fujiwara Y, Fukumoto M, et al. Unrecognized hepatic steatosis and non-alcoholic steatohepatitis in adjuvant tamoxifen for breast cancer patients. Oncol Rep. 2000;7(6):1299-304.

100. Hewitt KN, Pratis K, Jones ME, Simpson ER. Estrogen replacement reverses the hepatic steatosis phenotype in the male aromatase knockout mouse. Endocrinology. 2004;145(4):1842-8.

101. Fu X, Xing L, Xu W, Shu J. Treatment with estrogen protects against ovariectomy-induced hepatic steatosis by increasing AQP7 expression. Mol Med Rep. 2016;14(1):425-31.

102. Tarantino G, Valentino R, Di Somma C, D'Esposito V, Passaretti F, Pizza G et al. Bisphenol $A$ in polycystic ovary syndrome and its association with liver-spleen axis. Clin Endocrinol (Oxf). 2013;78(3):447-53.

103. Lee JM, Trauner M, Soroka CJ, Stieger B, Meier PJ, Boyer JL. Expression of the bile salt export pump is maintained after chronic cholestasis in the rat Gastroenterology. 2000;118(1):163-72.

104. Simon FR, Fortune J, Iwahashi M, Gartung C, Wolkoff A, Sutherland E. Ethinyl estradiol cholestasis involves alterations in expression of liver sinusoidal transporters. Am J Physiol. 1996;271(6 Pt 1):G1043-52.

105. Bhupathy P, Haines CD, Leinwand LA. Influence of sex hormones and phytoestrogens on heart disease in men and women. Womens Health (Lond). 2010;6(1):77-95.

106. Brady CW. Liver disease in menopause. World J Gastroenterol. 2015;21(25):7613-20.

107. Marino M, Galluzzo P, Ascenzi P. Estrogen signaling multiple pathways to impact gene transcription. Curr Genomics. 2006;7(8):497-508.

108. Chen J, Zhao KN, Liu GB. Estrogen-induced cholestasis: pathogenesis and therapeuticimplications. Hepatogastroenterology. 2013;60(126):1289-96.

109. Uebi T, Umeda M, Imai T. Estrogen induces estrogen receptor alpha expression and hepatocyte proliferation in the livers of male mice. Genes Cells. 2015;20(3):217-23.

110. Chico Y, Fresnedo O, Botham K, Lacort M, Ochoa B. Regulation of bile acid synthesis by estradiol and progesterone in primary cultures of rat hepatocytes. Exp Clin Endocrinol Diabetes. 1996;104(2):137-44.

111. Kushwaha RS, Born KM. Effect of estrogen and progesterone on the hepatic cholesterol 7-alpha-hydroxylase activity in ovariectomized baboons. Biochim Biophys Acta. 1991;1084(3):300-2.

112. Chico Y, Fresnedo O, Lacort M, Ochoa B. Effect of estradiol and progesterone on cholesterol 7 alpha-hydroxylase activity in rats subjected to different feeding conditions. Steroids. 1994;59(9):528-35.

113. Schreiber AJ, Simon FR. Estrogen-induced cholestasis: clues to pathogenesis and treatment. Hepatology. 1983;3(4):607-13.

114. Kontturi M, Sotaniemi E. Effect of oestrogen on liver function of prostatic cancer patients. Br Med J. 1969;4(5677):204-5.

115. Wang DQ, de Bari O, Wang HH. G protein-coupled receptor 30 (GPR30), a novel estrogen receptor, plays an independent role in the pathogenesis of estrogen-induced cholesterol gallstones in female mice. FASEB J. 2017.

116. Rizner TL, Penning TM. Role of aldo-keto reductase family 1 (AKR1) enzymes in human steroid metabolism. Steroids. 2014;79:49-63.

117. Thymiakou E, Zannis VI, Kardassis D. Physical and functional interactions between liver $X$ receptor/retinoid $X$ receptor and Sp1 modulate the transcriptional induction of the human ATP binding cassette transporter A1 gene by oxysterols and retinoids. Biochemistry. 2007;46(41):11473-83.

118. Rando G, Wahli W. Sex differences in nuclear receptor-regulated liver metabolic pathways. Biochim Biophys Acta. 2011;1812(8):964-73.

119. Williams ET, Leyk M, Wrighton SA, Davies PJ, Loose DS, Shipley GL, et al. Estrogen regulation of the cytochrome P450 3A subfamily in humans. J Pharmacol Exp Ther. 2004;311(2):728-35.

120. Hashimoto H, Toide K, Kitamura R, Fujita M, Tagawa S, Itoh S, et al. Gene structure of CYP3A4, an adult-specific form of cytochrome P450 in human livers, and its transcriptional control. Eur J Biochem. 1993;218(2):585-95.

121. Tang W, Norlin M, Wikvall K. Regulation of human CYP27A1 by estrogens and androgens in HepG2 and prostate cells. Arch Biochem Biophys. 2007; $462(1): 13-20$. 
122. Norlin M, Pettersson $H$, Tang W, Wikvall K. Androgen receptor-mediated regulation of the anti-atherogenic enzyme CYP27A1 involves the JNK/c-jun pathway. Arch Biochem Biophys. 2011;506(2):236-41.

123. DuSell CD, Umetani M, Shaul PW, Mangelsdorf DJ, McDonnell DP. 27hydroxycholesterol is an endogenous selective estrogen receptor modulator. Mol Endocrinol. 2008;22(1):65-77.

124. Tang W, Norlin M. Regulation of steroid hydroxylase CYP7B1 by androgens and estrogens in prostate cancer LNCaP cells. Biochem Biophys Res Commun. 2006;344(2):540-6.

125. Yamamoto Y, Moore R, Hess HA, Guo GL, Gonzalez FJ, Korach KS, et al. Estrogen receptor alpha mediates 17alpha-ethynylestradiol causing hepatotoxicity. J Biol Chem. 2006;281(24):16625-31.

126. Koopen NR, Post SM, Wolters H, Havinga R, Stellaard F, Boverhof R, et al. Differential effects of 17alpha-ethinylestradiol on the neutral and acidic pathways of bile salt synthesis in the rat. J Lipid Res. 1999:40(1):100-8

127. Johansson L, Thomsen JS, Damdimopoulos AE, Spyrou G, Gustafsson JA, Treuter E. The orphan nuclear receptor SHP inhibits agonist-dependent transcriptional activity of estrogen receptors ERalpha and ERbeta. J Biol Chem. 1999;274(1):345-53.

128. Lee YK, Dell H, Dowhan DH, Hadzopoulou-Cladaras M, Moore DD. The orphan nuclear receptor SHP inhibits hepatocyte nuclear factor 4 and retinoid $\mathrm{X}$ receptor transactivation: two mechanisms for repression. Mol Cell Biol. 2000;20(1):187-95

129. Gupta S, Stravitz RT, Dent P, Hylemon PB. Down-regulation of cholesterol 7alpha-hydroxylase (CYP7A1) gene expression by bile acids in primary rat hepatocytes is mediated by the c-Jun N-terminal kinase pathway. J Biol Chem. 2001;276(19):15816-22.

130. Bourdeau V, Deschenes J, Metivier R, Nagai Y, Nguyen D, Bretschneider $N$, et al. Genome-wide identification of high-affinity estrogen response elements in human and mouse. Mol Endocrinol. 2004;18(6):1411-27.

131. Legato MJ, Johnson PA, Manson JE. Consideration of sex differences in medicine to improve health care and patient outcomes. JAMA. 2016; 316(18):1865-6.

\section{Publisher's Note}

Springer Nature remains neutral with regard to jurisdictional claims in published maps and institutional affiliations.

Ready to submit your research? Choose BMC and benefit from:

- fast, convenient online submission

- thorough peer review by experienced researchers in your field

- rapid publication on acceptance

- support for research data, including large and complex data types

- gold Open Access which fosters wider collaboration and increased citations

- maximum visibility for your research: over $100 \mathrm{M}$ website views per year

At $\mathrm{BMC}$, research is always in progress.

Learn more biomedcentral.com/submissions 